\title{
Hypoglycemia unawareness in type 1 diabetes suppresses brain responses to hypoglycemia
}

\author{
Janice Jin Hwang, ${ }^{1}$ Lisa Parikh, ${ }^{1}$ Cheryl Lacadie, ${ }^{2}$ Dongju Seo, ${ }^{3}$ Wai Lam, ${ }^{1}$ Muhammad Hamza, ${ }^{1}$ Christian Schmidt, ${ }^{1}$ Feng Dai, ${ }^{4}$ \\ Anne-Sophie Sejling, ${ }^{5}$ Renata Belfort-DeAguiar, ${ }^{1}$ R. Todd Constable, ${ }^{2}$ Rajita Sinha, ${ }^{3}$ and Robert Sherwin ${ }^{1}$ \\ 'Section of Endocrinology, ${ }^{2}$ Department of Radiology \& Biomedical Imaging, and ${ }^{3}$ Department of Psychiatry, Yale School of Medicine, New Haven, Connecticut, USA. ${ }^{4}$ Yale Center for Analytical Sciences, \\ Yale School of Public Health, New Haven, Connecticut, USA. '5Department of Cardiology, Nephrology and Endocrinology, Nordsjællands Hospital, Hillerød, Denmark.
}

BACKGROUND. Among nondiabetic individuals, mild glucose decrements alter brain activity in regions linked to reward, motivation, and executive control. Whether these effects differ in type 1 diabetes mellitus (T1DM) patients with and without hypoglycemia awareness remains unclear.

METHODS. Forty-two individuals (13 healthy control [HC] subjects, 16 T1DM individuals with hypoglycemia awareness [T1DM-Aware], and 13 T1DM individuals with hypoglycemia unawareness [T1DM-Unaware]) underwent blood oxygen leveldependent functional MRI brain imaging during a 2-step hyperinsulinemic euglycemic ( $90 \mathrm{mg} / \mathrm{dl})$-hypoglycemic (60 mg/dl) clamp for assessment of neural responses to mild hypoglycemia.

RESULTS. Mild hypoglycemia in HC subjects altered activity in the caudate, insula, prefrontal cortex, and angular gyrus, whereas T1DM-Aware subjects showed no caudate and insula changes, but showed altered activation patterns in the prefrontal cortex and angular gyrus. Most strikingly, in direct contrast to HC and T1DM-Aware subjects, T1DM-Unaware subjects failed to show any hypoglycemia-induced changes in brain activity. These findings were also associated with blunted hormonal counterregulatory responses and hypoglycemia symptom scores during mild hypoglycemia.

CONCLUSION. In T1DM, and in particular T1DM-Unaware patients, there is a progressive blunting of brain responses in cortico-striatal and fronto-parietal neurocircuits in response to mild-moderate hypoglycemia. These findings have implications for understanding why individuals with impaired hypoglycemia awareness fail to respond appropriately to falling blood glucose levels.

FUNDING. This study was supported in part by NIH grants R01DK020495, P30 DK045735, K23DK109284, K08AA023545. The Yale Center for Clinical Investigation is supported by an NIH Clinical Translational Science Award (UL1 RR024139).

\section{Introduction}

Patients with type 1 diabetes mellitus (T1DM) have long been constrained by the adverse effects of insulin-induced hypoglycemia. The Diabetes Control and Complications Trial (DCCT) established the benefits of restoring mean blood glucose to nearnormal levels in patients with T1DM, and while this has produced clear benefits in terms of the microvascular and macrovascular complications of T1DM, for many individuals, the widespread use of intensified insulin therapy has resulted in a much higher rate of severe hypoglycemia (1). Frequent episodes of hypoglycemia can lead to hypoglycemia unawareness, which prevents patients from taking corrective action by eating. Thus, for many T1DM

Role of funding source: The funding agencies had no role in the design and conduct of the study; collection, management, analysis, and interpretation of the data; or the preparation, review, or approval of the manuscript.

Conflict of interest: JJH and R. Sherwin report receipt of research support from Regeneron. RBD reports receipt of research support from Glaxo Smith Kline.

Submitted: September 26, 2017; Accepted: January 23, 2018.

Reference information: / Clin Invest. 2018;128(4):1485-1495

https://doi.org/10.1172/JCl97696. patients the immediate fear of hypoglycemia exceeds the fear of long-term complications $(2,3)$.

In nondiabetic subjects, hypoglycemia is rare because, in response to falling blood glucose levels, an integrated physiologic response is triggered that suppresses endogenous insulin secretion, increases release of counterregulatory hormones, and provokes awareness of hypoglycemia, which act together to rapidly restore euglycemia by stimulating glucose production and food consumption. We have previously reported using the glucose clamp technique together with functional magnetic resonance (fMRI) imaging, visual food cues, and behavioral measures that brain regions involved in stimulating motivation to eat are exquisitely sensitive to small reductions in glucose. In healthy humans, mild reductions in plasma glucose $(\sim 68 \mathrm{mg} / \mathrm{dl})$ that were not sufficient to increase counterregulatory hormones were sufficient to activate hypothalamic blood flow (4) as well as modulate brain motivation/reward and executive control responses to food cues, which in turn resulted in a greater craving for high-calorie foods (5).

In T1DM, this critical hypoglycemia defense system may be interrupted at every level. Loss of endogenous insulin and reliance on peripheral exogenous hormone delivery make rapid insulin 
Table 1. Participant characteristics

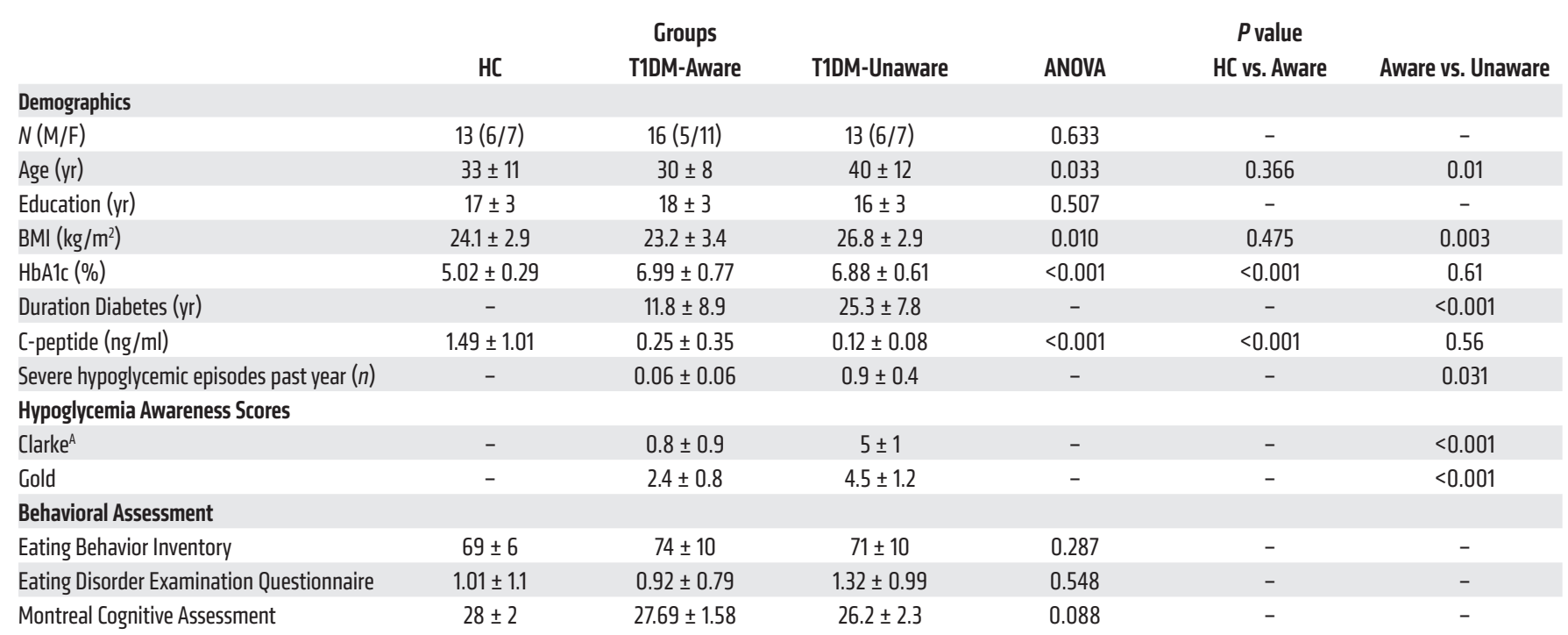

${ }^{A}$ Clarke score, $\leq 2 \mathrm{R}$ = hypoglycemia aware; $\geq 4 \mathrm{R}$ = hypoglycemia unaware. Data presented as the mean \pm SEM.

reductions impossible. $\beta$ Cell destruction is also linked to loss of glucagon responses to hypoglycemia, a defect that develops in nearly all T1DM patients $(6,7)$. As a result, T1DM patients are particularly vulnerable to impairments in epinephrine release, which commonly follows iatrogenic insulin-induced hypoglycemia (8-10).

Frequent episodes of hypoglycemia in T1DM individuals commonly lead to hypoglycemia-associated autonomic failure (HAAF), whereby significantly lower blood glucose levels are required to elicit a counterregulatory hormonal response as well as symptomatic awareness of hypoglycemia $(2,3,9)$. Whether loss of hypoglycemia awareness is also accompanied by a failure to activate the drive to eat, which is clinically the most effective way to reverse hypoglycemia, remains unknown. A study using fMRI reported that functional connectivity in brain regions that have been implicated in the control of feeding behavior including the basal ganglia, insula, and prefrontal cortex are altered in individuals with T1DM (11). However, this study did not examine the specific effects of HAAF and hypoglycemia unawareness on brain activity. Another study in a small number of individuals with T1DM who were both aware or unaware of hypoglycemia using $\left[{ }^{18} \mathrm{~F}\right]$ fluoro-2-deoxyglucose (FDG) PET scanning suggested that acute hypoglycemia may increase ventral striatum FDG uptake and that a small diminution of this response may have occurred in unaware patients (12). However, FDG uptake may not accurately reflect glucose uptake during hypoglycemia, since acute hypoglycemia (and likely antecedent hypoglycemia) alters the lumped constant used to calculate glucose uptake (13).

It is noteworthy that prior studies using fMRI or PET scanning to assess the impact of hypoglycemia on the brain among T1DM individuals have utilized very low glycemic targets (typically 50 $\mathrm{mg} / \mathrm{dl}$ or less). However, from a clinical perspective, by the time a T1DM patient's blood glucose falls into the low $50 \mathrm{mg} / \mathrm{dl}$ range they may already be at significantly higher risk for hypoglycemiaassociated morbidity and mortality due to the failure to appropri- ately activate multiple layers of protection against hypoglycemia. Therefore, in this study, we specifically sought to determine how T1DM individuals with or without hypoglycemia unawareness respond to milder degrees of hypoglycemia in an effort to more effectively distinguish the CNS defects at an earlier time point leading to unawareness in the course of developing moderatesevere hypoglycemia. To do this, we used fMRI brain scanning in conjunction with a 2-step hyperinsulinemic euglycemichypoglycemic clamp technique to investigate how regional brain activity, particularly the neurocircuits driving eating behavior, are altered among T1DM individuals with hypoglycemia unawareness (T1DM-Unaware) as compared with those patients with preserved awareness (T1DM-Aware) as well as healthy nondiabetic control (HC) subjects during acute mild-moderate hypoglycemia (target $\sim 60 \mathrm{mg} / \mathrm{dl}$ ).

\section{Results}

\section{Participant characteristics}

Thirteen HC individuals, 16 T1DM-Aware individuals as assessed by the Clarke score (14), and 13 T1DM-Unaware individuals participated in this study. Demographic and clinical characteristics are presented in Table 1. Compared with HC individuals, both T1DMAware individuals and T1DM-Unaware individuals were similar in age, gender, and education. The T1DM-Unaware individuals were slightly older $(P=0.01)$, had longer duration of disease $(P<$ $0.001)$, and had slightly higher BMI $(P=0.003)$ than the T1DMAware group. T1DM-Unaware individuals also had significantly higher self-reported rates of severe hypoglycemic episodes in the preceding year $(P=0.03)$. Both T1DM-Aware and T1DM-Unaware groups were indistinguishable in terms of percentage glycated hemoglobin (HbA1c), and there were no differences across all 3 groups for gender and education as well as measures of disordered eating and cognitive function (Table 1). 


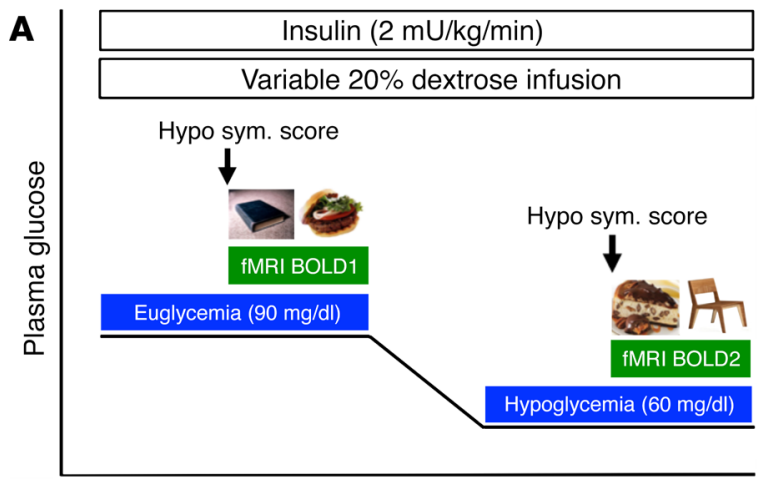

B

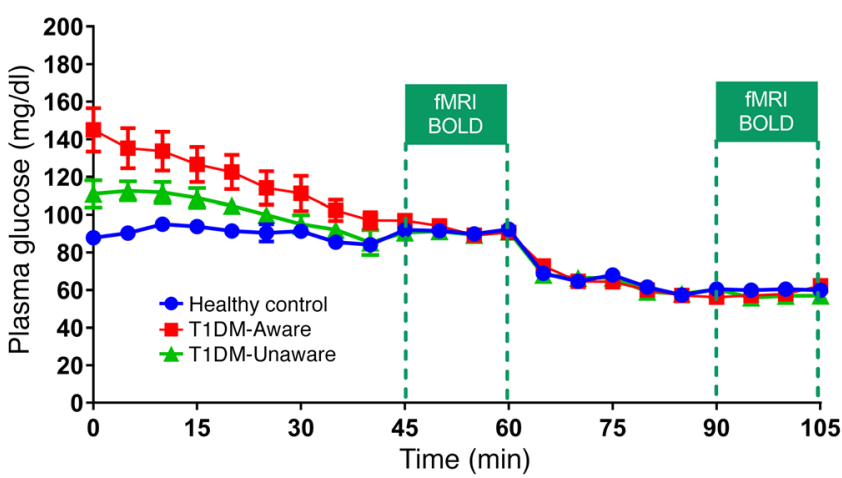

Figure 1. Study design. (A) Schematic representation of 2-step hyperinsulinemic euglycemic-hypoglycemia clamp during fMRI BOLD scanning in response to visual cues. (B) Plasma glucose levels of healthy control $(n=13)$, T1DM-Aware $(n=16)$, and T1DM-Unaware $(n=13)$ during the study. Data presented as the mean \pm SEM. Statistical comparisons were performed using mixed-model linear regression adjusting for age, gender, and BMI.

\section{Two-step hyperinsulinemic euglycemic-hypoglycemic clamp}

As seen in Figure 1B, both groups of individuals with T1DM had modestly higher blood glucose levels at the beginning of the study compared with HC subjects. However, using repeated-measures linear regression analysis and adjusting for age, BMI, and gender, there were no overall differences in plasma glucose levels during the course of the study between T1DM-Aware and T1DMUnaware subjects (least squares mean 5.3 [95\% CI -4.1, 14.7], $P=$ 0.27). Furthermore, beginning at 25 minutes, there was no statistically significant difference in pair-wise comparisons of plasma glucose levels between T1DM-Unaware compared with T1DMAware $(P=0.11)$ as well as HC participants $(P=0.14)$. Notably, during the times of fMRI blood oxygen level-dependent (BOLD) data acquisition (euglycemia at 45-60 minutes and hypoglycemia at 90-105 minutes), plasma glucose levels were virtually identical across all 3 groups and were at target (mean plasma glucose at euglycemia $93.0 \pm 1.9 \mathrm{mg} / \mathrm{dl}$ and hypoglycemia $58.9 \pm 1.1 \mathrm{mg} / \mathrm{dl}$ ) (Figure 1B). There were also no differences in plasma insulin levels between the groups $(P=0.76)$ over time. In addition, there were no significant differences across groups in mean glucose infusion rates (GIRs) during euglycemia (GIR [mg/kg/min], HC $9.5 \pm 1.1$ vs. T1DM-Aware $8.2 \pm 1.4$ vs. T1DM-Unaware $7.0 \pm 0.9, P=0.35$ ) as well as during hypoglycemia (HC $7.2 \pm 0.8$ vs. T1DM-Aware 6.6 \pm 0.7 vs. T1DM-Unaware $4.9 \pm 0.8, P=0.12$.

\section{Hormonal and symptomatic responses to hypoglycemia}

Mean plasma epinephrine, norepinephrine, glucagon, and cortisol levels at euglycemia and hypoglycemia are shown in Figure 2. Baseline epinephrine levels were different across groups (ANOVA $P=0.04)$. This difference was primarily driven by the difference between T1DM-Unaware compared with HC participants $(P=$ 0.024). T1DM-Unaware and T1DM-Aware patients were not significantly different $(P=0.07)$. Notably, plasma epinephrine levels rose significantly in response to hypoglycemia in all 3 groups. HC and T1DM-Aware subjects had a nearly 3-fold increase in epinephrine levels, whereas T1DM-Unaware individuals had a much more modest response, i.e., only a $60 \%-70 \%$ increase above euglycemic levels. In contrast, only the HCs had a significant increase in plasma glucagon and cortisol during the hypoglycemic phase of the study. No significant changes in plasma norepinephrine were detected in the 3 groups during this relatively mild hypoglycemic stimulus.

While in the scanner and prior to the fMRI BOLD acquisitions (at 30 and 75 minutes), participants were asked to rate their symptoms of hypoglycemia using the Edinburgh hypoglycemia score (15). Both T1DM-Aware and HC subjects exhibited a statistically significant increase in symptom response during hypoglycemia, whereas there was no significant change in symptoms in the T1DM-Unaware group (Figure 3). Interestingly, hypoglycemia symptoms were different across groups during hypoglycemia (HC, $23.9 \pm 7.0$; T1DM-Aware, $35.9 \pm 14.2$; T1DM-Unaware, $28.4 \pm 12.4$; ANOVA $P=0.03$ ), with the highest scores among T1DM-Aware individuals, which was due to differences between $\mathrm{HC}$ and T1DMAware individuals $(P=0.009)$. Furthermore, during hypoglycemia, the symptoms of hypoglycemia correlated significantly and positively with plasma epinephrine levels for the T1DM-Aware individuals $(\rho=0.58, P=0.02)$, but not for T1DM-Unaware $(P=$ 0.54). Of note was that one participant who was T1DM-Unaware based on Clarke score had a very pronounced increase in plasma epinephrine levels during mild hypoglycemia (euglycemia $47 \mathrm{pg} /$ $\mathrm{ml}$, peak hypoglycemia $329 \mathrm{pg} / \mathrm{ml}$ ); however, this participant had minimal changes in hypoglycemia symptom scores during acute hypoglycemia (Edinburgh score euglycemia 33, hypoglycemia 39), despite achieving target glucose levels during both the euglycemic and hypoglycemia portions of the clamp. As a result, all fMRIbased analyses were run with and without this participant. Given that there were no significant changes in the results, this participant was included in all subsequent analyses.

\section{Neural responses to mild hypoglycemia}

Overall relationship between groups and glycemia (group $\times$ condition effects). Across all 3 groups, there was a significant group difference in brain response to hypoglycemia and euglycemia in the right striatum (dorsal/ventral), particularly in the caudate (Figure 4) even after adjusting for age and BMI and using the current standard threshold for significance of $P$ less than 0.001 (whole brain, family- 


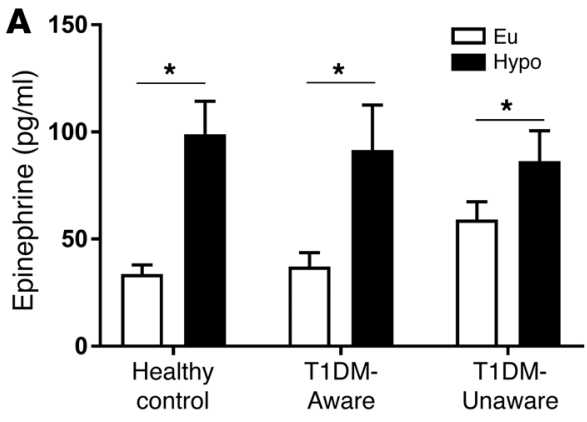

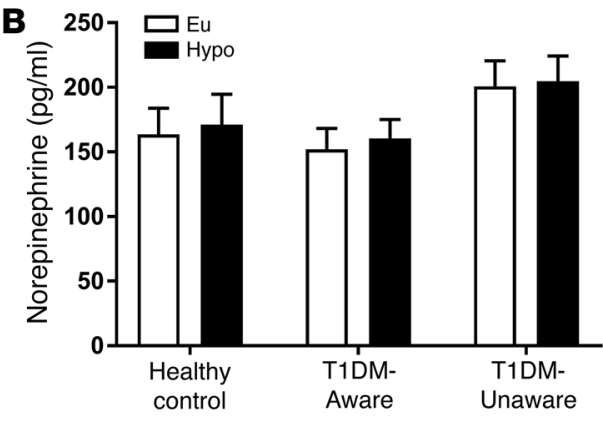

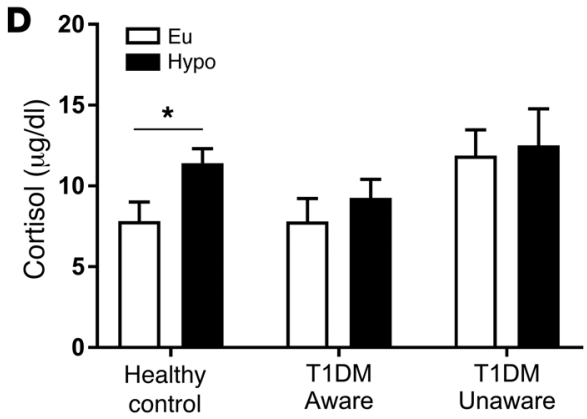

control

Aware
Figure 2. Hormonal responses to mild hypoglycemia in healthy control $(n=13)$, T1DM-Aware $(n=16)$, and T1DM-Unaware ( $n=13$ ) during the study. (A) Epinephrine, (B) norepinephrine, (C) glucagon, (D) cortisol. Open bars denote euglycemia, black bars denote hypoglycemia. Euglycemia values were averaged from those obtained at 45-60 minutes of clamp. Hypoglycemia values were averaged from those obtained at 90-105 minutes of clamp. Data presented as the mean \pm SEM. ${ }^{*} P<0.05$ by paired samples $t$ test.

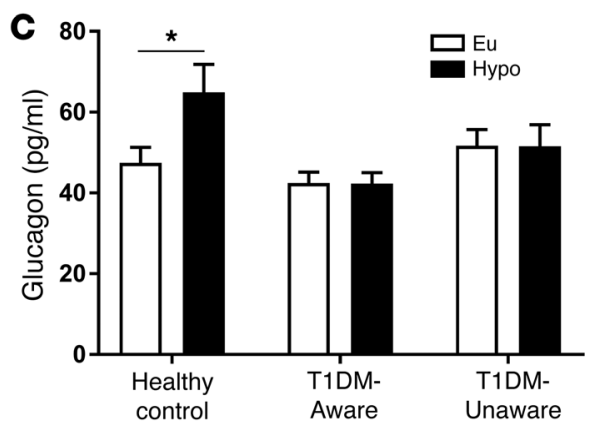

wise error [FWE] corrected) (16). To give a sense of directionality of change, a region of interest was defined from the significant cluster in the right caudate and mean general linear model (GLM) $\beta$-weights were extracted for each subject. In response to hypoglycemia, HC subjects had relatively decreased activity in the caudate, whereas T1DM-Aware and T1DM-Unaware individuals had minimal changes (Figure 4B). Across all 3 groups, we did not find any significant (at $P<0.001$ ) interactions between group $\times$ glycemia $\times$ task or group $\times$ task. Thus, all analyses using all 3 groups were collapsed across tasks (visual food and non-food cues). Furthermore, although all 3 groups had similar plasma glucose levels by 20 minutes prior to the time of BOLD acquisitions, the T1DM-Aware group had higher plasma glucose levels at the start of the clamps. To assess whether these differences in starting glucose levels affected brain activity during euglycemia BOLD acquisitions ( $\sim 5$ minutes later), we assessed across-group and between-group interactions at euglycemia alone and found no significant differences.

We then examined each group's brain activity response to hypoglycemia separately (Figure 5 and Supplemental Table 1; supplemental material available online with this article; https:// doi.org/10.1172/JCI97696DS1). The HC, T1DM-Aware, and T1DM-Unaware subjects had strikingly different patterns of brain responses to mild hypoglycemia, even after adjusting for age and BMI. In particular, in response to hypoglycemia, HC subjects had relatively decreased activity in the ventral striatum/caudate, insula, orbitofrontal cortex (OFC), ventromedial prefrontal cortex (vmPFC), ventrolateral prefrontal cortex (vlPFC), dorsolateral prefrontal cortex (dlPFC), and left angular gyrus. In contrast, while the T1DM-Aware individuals also had relatively decreased activity in the VmPFC and OFC, they did not have any significant differences in activity in the caudate, insula, or dlPFC. Interestingly, the T1DM-Aware individuals had relatively increased activity in the inferior parietal lobe, particularly the right angular gyrus as well as the right vlPFC. In contrast, T1DM-Unaware individu- als showed no significant changes in brain activity in any of the regions that were different among the other 2 groups.

Given that changes in plasma epinephrine levels are believed to be a particularly sensitive marker for defective counterregulation among T1DM individuals, we assessed the relationship between changes in plasma epinephrine levels and changes in brain responses in the regions identified in Figure 5. A smaller change in plasma epinephrine levels was associated with a smaller degree of deactivation in the striatum/caudate $(\rho=-0.43, P=$ $0.005), \operatorname{vmPFC}(\rho=-0.46, P=0.003)$, right insula $(\rho=-0.37, P=$ $0.02)$, vlPFC $(\rho=-0.34, P=0.03)$, and angular gyrus $(\rho=-0.41, P=$ 0.007), consistent with the association of the blunted epinephrine response with the blunted brain responses. There were no associations between brain activity in any of the above regions and epinephrine levels at euglycemia or hypoglycemia alone. Among the T1DM subjects, the Edinburgh hypoglycemia symptom score

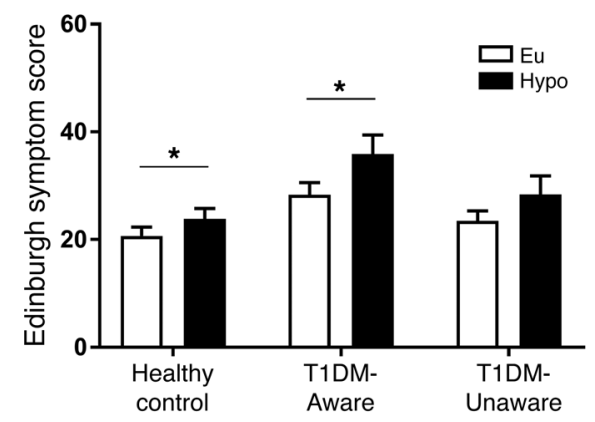

Figure 3. Hypoglycemia symptom scores in healthy control $(n=13)$, T1DM-Aware $(n=16)$, and T1DM-Unaware $(n=13)$. Symptoms of hypoglycemia from the Edinburgh hypoglycemia symptom score were administered on a Likert scale (1-7) and results were summed. Open bars denote euglycemia, black bars denote hypoglycemia. Data presented as the mean \pm SEM. ${ }^{*} P<0.05$ by paired samples $t$ test. 


\section{A}

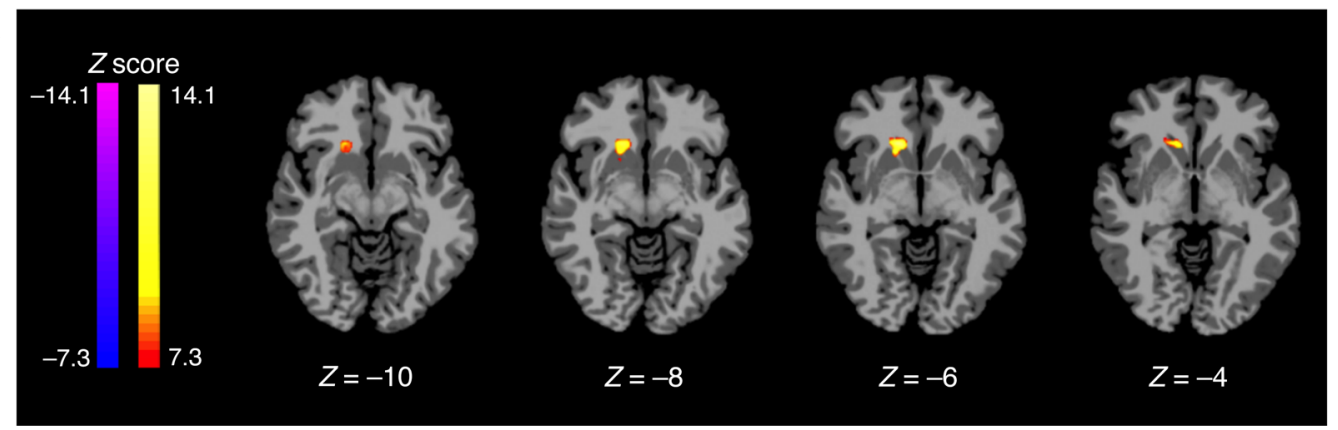

B
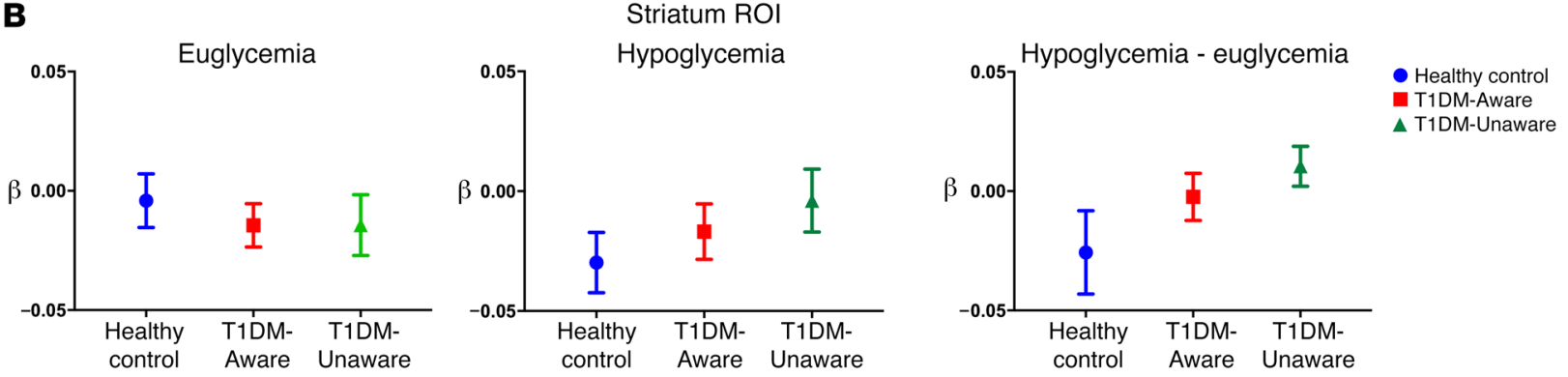

Figure 4. Group $\times$ glycemia effects. (A) Axial slices showing the differences in brain responses to mild hypoglycemia compared with euglycemia across all 3 groups (covaried for age and BMI, threshold of $P<0.001,2$-tailed, FWE whole-brain corrected). (B) Region of interest (ROI) identified from significant cluster in right striatum (caudate). General linear model $\beta$-weights extracted from each participant, presented as mean $(95 \% \mathrm{Cl})$. Healthy control $n=13$, T1DM-Aware $n=16$, T1DM-Unaware $n=13$.

correlated inversely with activity only in the vmPFC during hypoglycemia $(\rho=-0.410, P=0.03)$.

\section{Effect of hypoglycemia unawareness on brain responses to high-calorie food cues}

To address the question of whether hypoglycemia unawareness modulates the brain's response to hypoglycemia while viewing high-calorie food stimuli, we performed an analysis focused on only the T1DM-Aware and T1DM-Unaware groups. While viewing high-calorie food cues ( $75 \%$ of the high-calorie cues were also high carbohydrate), there was a significant group $\times$ glycemia interaction $(P<0.001)$, even after covarying for age, BMI, and duration of diabetes. This interaction was not present under non-food visual stimuli conditions. Notably, T1DM-Aware individuals had a significant decrease in brain activity during high-calorie food in the medial OFC (Brodmann area 11), while T1DM-Unaware individuals showed no statistically significant change in brain activity in this region (Figure 6). There were no significant correlations between brain activity in this region and counterregulatory hormones.

\section{Discussion}

In this study, we show that hypoglycemia unawareness in T1DM patients is associated with a diminished brain response to mild hypoglycemia (plasma glucose $\sim 60 \mathrm{mg} / \mathrm{dl}$ ). Moreover, the pattern of loss of brain responses appears to involve cortico-striatal and fronto-parietal neurocircuits that are known to play important roles in regulating motivation and goal-directed behavior as well as attention, and thus are likely to have implications for understanding why individuals with hypoglycemia unawareness fail to respond appropriately to falling blood glucose levels.
The basal ganglia, and in particular the caudate, has been consistently shown in studies across species and imaging modalities to play an important role in the ability to respond appropriately to environmental changes and to regulate goal-directed behavioral inputs (17-21). The caudate has direct physical and functional connections with executive control regions in the frontal cortex including the medial, ventral, and dorsolateral PFC $(22,23)$. Among HC individuals, mild hypoglycemia was sufficient to elicit changes in the caudate, cortical regions such as the vmPFC and vlPFC, and the insula, which is consistent with previous studies that have shown that the caudate, PFC, and insula are responsive to changes in circulating glucose levels $(5,12,24,25)$. In contrast, T1DM-Aware individuals had altered patterns of cortico-striatal activity with no significant changes in the caudate or insula during hypoglycemia. We also observed differences across groups in the patterns of activation/deactivation in the dlPFC and angular gyrus. The angular gyrus, located in the inferior parietal lobe, has direct projections to the dIPFC (26) and together they are part of a larger, well-studied, fronto-parietal circuit (27-29). The angular gyrus, in particular, has been shown to play a role in regulating how one's attention shifts towards higher salient stimuli (30-33). Interestingly, among $\mathrm{HC}$ subjects, mild hypoglycemia induced changes in activity in the left dlPFC and left angular gyrus, which is consistent with a previous study in HC subjects during hypoglycemia (plasma glucose $50 \mathrm{mg} / \mathrm{dl}$ ) while performing cognitive tasks (34). In contrast, T1DM-Aware individuals had no brain responses in the left dlPFC or left angular gyrus, but instead showed markedly increased activity in the right angular gyrus. Taken together with our findings that T1DM-Aware individuals had higher ratings for symptoms at hypoglycemia, these observations suggest 


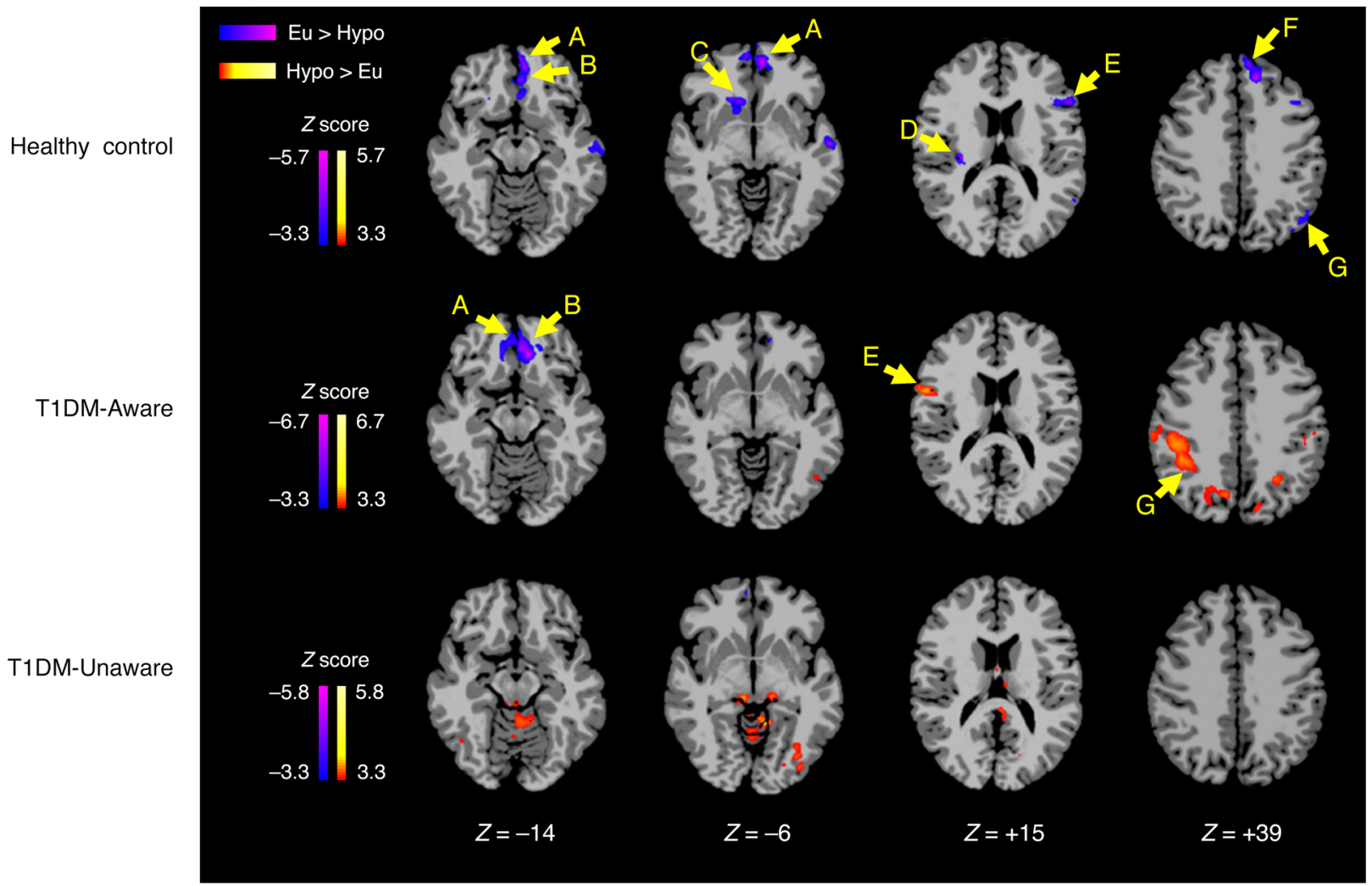

Figure 5. Differences in regional brain responses between mild hypoglycemia and euglycemia conditions. Axial slices of healthy control ( $n=13)$, T1DM-Aware $(n=16)$, and T1DM-Unaware $(n=13)$ subjects showing differences in brain responses to mild hypoglycemia (Hypo-Eu) (covaried by age and BMI, threshold of $P<0.001$, 2-tailed, FWE whole-brain corrected). Regions identified as: $\mathrm{A}=$ ventromedial prefrontal cortex (vmPFC); $\mathrm{B}=$ orbitofrontal cortex (OFC); $\mathrm{C}=$ right ventral striatum/caudate; $\mathrm{D}=$ right insula; $\mathrm{E}=$ ventrolateral prefrontal cortex (vIPFC); $\mathrm{F}=$ dorsolateral prefrontal cortex (dIPFC); $\mathrm{G}=$ angular gyrus.

that increased activity in inferior parietal lobe/angular gyrus may be a compensatory adaptation to the disruption in cortico-striatal and fronto-parietal neurocircuits that are involved in sensing mild hypoglycemia. The markedly increased angular gyrus activity seen in the T1DM-Aware group during mild hypoglycemia may reflect differences in attention to or sensing of the stimulus (35). Thus, the T1DM-Aware individuals may have heightened awareness to hypoglycemia sensory inputs compared with HC subjects, which would be consistent with their higher reported ratings of hypoglycemia symptoms both at euglycemia and at hypoglycemia.

Most strikingly, compared with T1DM-Aware and HC subjects, the T1DM-Unaware participants showed virtually no changes in brain activity in response to mild hypoglycemia. Very little is known about the impact of hypoglycemia unawareness on regional brain responses; however, these findings would be consistent with the blunted symptom scores as well as the blunted counterregulatory hormone responses to hypoglycemia observed in the T1DM-Unaware group. The underlying mechanism mediating the lack of change among the T1DM-Unaware individuals remains uncertain; however, it is likely due to brain adaptations to frequent episodes of severe hypoglycemia in the preceding year of the study. Recurrent hypoglycemia alters brain glucose transport kinetics as well as promotes increased utilization of alternate fuels such as monocarboxylic acids (lactate, ketones, and acetate) in humans when the availability of glucose diminishes $(36,37)$.
Furthermore, T1DM individuals with hypoglycemia unawareness may have alterations in cerebral blood flow during hypoglycemia $(38,39)$, which may also affect BOLD signal. Interestingly, a recent study has reported that individuals with T1DM and hypoglycemia unawareness have increased cerebral blood flow during acute hypoglycemia compared with T1DM-Aware and HC subjects (39). The current findings would be consistent with these observations that the brain adapts to ensure sufficient substrate (glucose) delivery to the brain. In keeping with these human studies, data in rodents have also demonstrated that prior exposure to hypoglycemia induces upregulation of blood-brain-barrier glucose transport, leading to more efficient glucose utilization during hypoglycemia $(40,41)$. Thus, the lack of change in brain activity among T1DM-Unaware individuals in response to mild hypoglycemia may be the culmination of a variety of adaptive changes in cerebral blood flow, glucose transport, cerebral glucose metabolism, or some combination of each of these factors.

It is important to note that induction of hypoglycemia results in a series of dynamic changes in brain activation and deactivation, and thus time intervals when the scans are acquired over the course of hypoglycemia may directly impact the directionality and regional changes observed (24). This, as well as other factors such as hypoglycemia target, timing of image acquisition, and imaging modality, may all contribute to the heterogeneity of brain responses to hypoglycemia previously reported in the literature. For 

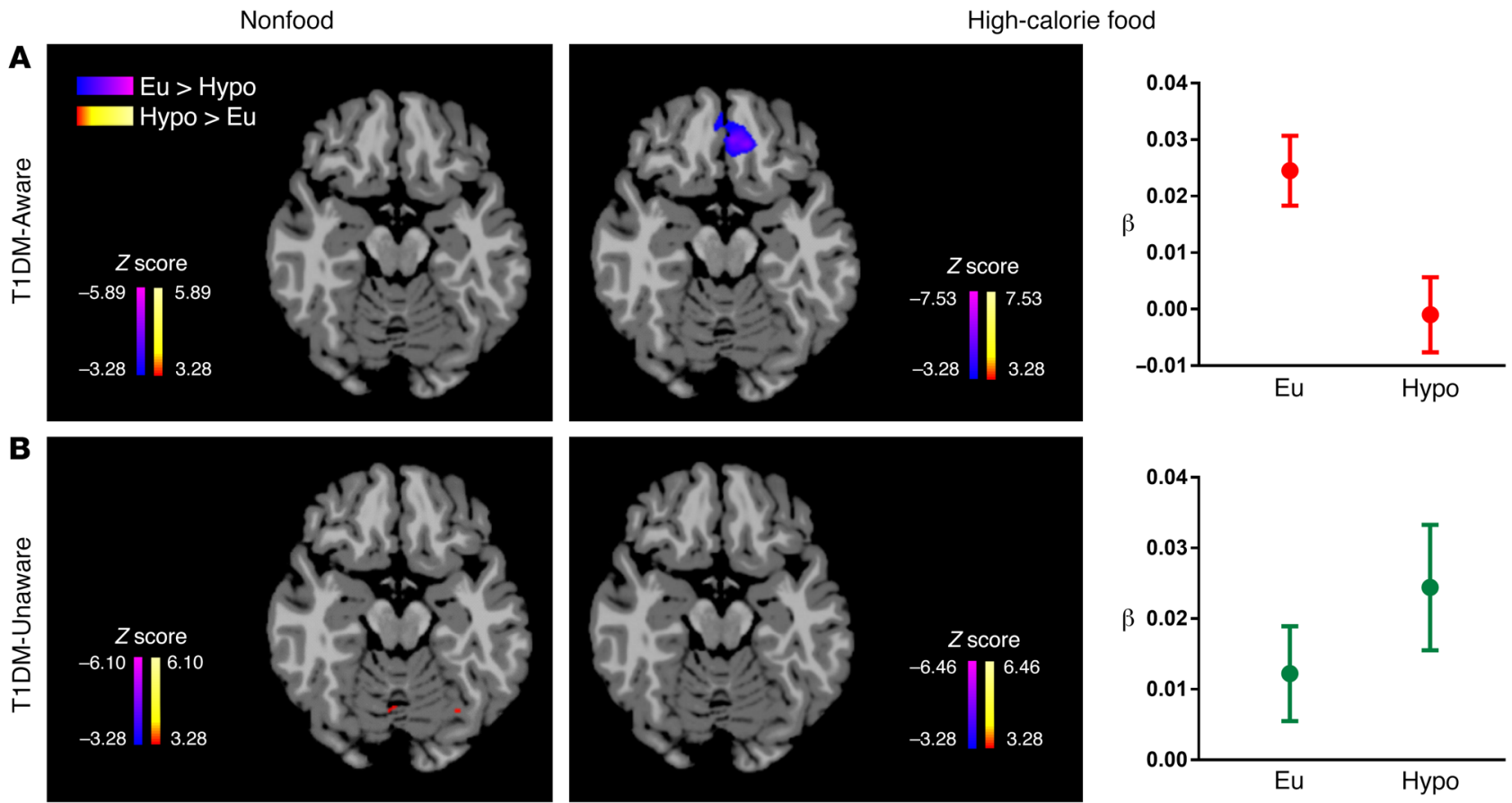

Figure 6. Brain responses to high-calorie food cues. Axial brain slices $(Z=-17)$ of change in brain response to mild hypoglycemia (Hypo - Eu) while viewing high-calorie food cues and non-food cues in (A) T1DM-Aware $(n=16)$ and (B) T1DM-Unaware $(n=13)$ participants (covaried by age, duration of diabetes, and $\mathrm{BMI}$, threshold of $P<0.001$, 2-tailed, FWE whole-brain corrected). Data presented as the mean ( $95 \% \mathrm{Cl})$.

example, we did not observe hypoglycemia-induced changes in the hypothalamus, which has been reported by some groups (25), but not others (42) to be altered during hypoglycemia in T1DM individuals. Thus, our findings must be interpreted cautiously given that we are only observing a snapshot of the dynamic brain changes produced over the course of falling blood glucose levels, a critical time for prevention of hypoglycemia-induced brain injury.

Importantly, it remains uncertain whether lower glycemic thresholds will be able to elicit changes in brain activation responses among T1DM-Unaware individuals and whether the brain responses will be in a similar pattern to that observed among T1DM-Aware individuals. Studies of glucose transport kinetics in hypoglycemia T1DM-Unaware individuals have found that glucose transport is preserved even at glucose levels as low as 50 $\mathrm{mg} / \mathrm{dl}$ (43). However, it remains uncertain whether lower glucose thresholds are the only difference between T1DM-Aware and -Unaware individuals. Thus, this current study highlights the need for further studies designed to assess the contribution of additional factors such as age of onset of diabetes, duration of diabetes, and severity of diabetes during childhood/adolescence when the brain is still developing in determining the propensity for developing hypoglycemia unawareness. Furthermore, whether these changes are reversible and whether strict avoidance of hypoglycemia can restore brain responses remains to be assessed. Of note, prior studies using strict avoidance of hypoglycemia have also resulted in worsening of glycemic control (44-46), which could also have an impact on glucose transport capacity into the brain.

Because one of the earliest and best defenses against falling blood glucose levels is to eat, we also examined the brain responses to high-calorie/high-carbohydrate food cues. Among nondiabetic individuals, high-calorie food cues have been shown to elicit robust changes in brain activity in reward, motivation, and executive control regions during both euglycemia (47) and mild hypoglycemia (5). Consistent with these findings reported in nondiabetic individuals, the current data demonstrate that T1DM-Aware individuals also had a pronounced change in the medial OFC when viewing highcalorie food cues that was not present when looking at pictures of non-food objects. Notably, the medial OFC plays an important role in reward-guided decision making $(48,49)$. Furthermore, because it has dense direct connections with the hypothalamus (50,51), it has been shown to play a particularly important role in regulating feeding behavior (52-54). Thus, it is particularly noteworthy that in contrast to T1DM-Aware individuals, high-calorie food cues had no effect on medial OFC brain activity during mild hypoglycemia in T1DM-Unaware individuals, suggesting a diminished drive to eat, which may be a critical early defect in the defense against hypoglycemia. Interestingly, we found no relationship between changes in brain activity to high-calorie foods and the counterregulatory hormone response. Whether the lack of brain response is due to intrinsic CNS differences or secondary to the blunted rise in circulating counterregulatory hormone levels remains unclear and further studies will be needed to address this question and prove causality. However, given that in nondiabetic subjects changes in brain activity induce and occur prior to changes in counterregulatory hormones (4), it is likely that changes in brain activity are not primarily driven by the counterregulatory response, but rather play the key role in protecting the brain by initiating appropriate defenses against falling glucose levels. Prior studies have also noted a dissociation between counterregulatory hormone responses and awareness of hypoglycemia (45). 
It is noteworthy that there are some considerations and limitations to the current study. While we defined our groups using widely accepted and validated questionnaires for hypoglycemia unawareness, the Clarke and Gold scores, these are subjective reports and we did not collect data on glycemic variability and objective rates of hypoglycemia in the months preceding our studies. In addition, our T1DM-Unaware participants were approximately 10 years older and had diabetes for a longer duration than the T1DM-Aware group. Although we covaried for age, BMI, and duration of diabetes, our findings among the T1DM-Unaware individuals should still be interpreted cautiously with recognition that it may be very difficult experimentally to separate the effects of age and longer duration of T1DM from the effects of hypoglycemia unawareness itself. Of note in this regard, increasing age has been associated with increases in baseline epinephrine levels (55) and our T1DM-Unaware cohort was slightly older and had higher baseline epinephrine levels; however, we did not observe any relationships between epinephrine levels at euglycemia or hypoglycemia and brain responses. Furthermore, prior studies have examined the effects of age on counterregulatory responses to hypoglycemia (among nondiabetic individuals). In these studies, where the mean age of the older groups was markedly older than our cohort (age 60-70s), they found modest (55) or no (56) differences in counterregulatory responses to hypoglycemia.

It is also noteworthy that increased age and duration of diabetes may be associated with cerebrovascular dysfunction. Increased presence of cerebral small vessel disease such as white matter hyperintensities and lacunes have been reported among individuals with T1DM (mean age 50 years) $(57,58)$; however, other studies among older T1DM patients (mean age $\sim 60$ years and with known microvascular complications) (59) have reported no significant differences in white matter lesions or microinfarcts compared with control subjects. While we cannot exclude the possibility that occult cerebrovascular disease may also contribute to the differences observed in the T1DM-Unaware individuals, this appears less likely given our participants had well-controlled diabetes, had no history of cerebrovascular disease or cardiovascular disease, and were significantly younger (mean age 30 and 40 years for T1DM-Aware and -Unaware, respectively) than the groups reported in the literature.

Finally, even though our study includes larger numbers of T1DM-Aware and T1DM-Unaware participants than prior fMRIbased studies investigating hypoglycemia unawareness, our sample sizes remain a limitation. To minimize the risk of false positives, we used a $P$-value threshold of less than 0.001 (16). Currently, best practice guidelines for conducting fMRI based studies typically recommend at least 20 subjects per group to minimize false positives (60); however, these recommendations may not be directly applicable to studies among relatively rare disease groups such as individuals with T1DM and hypoglycemia unawareness or in study designs using highly controlled physiologic manipulations such as in a 2-step euglycemic-hypoglycemic clamp where individuals are compared to themselves at 2 well-defined, but different states.

In conclusion, the current study highlights the differential CNS responses to mild hypoglycemia among individuals with T1DM and preserved or diminished hypoglycemia awareness. Our findings suggest that although T1DM-Aware individuals no longer exhibit hypoglycemia-induced changes in reward and motivation brain regions (striatum), they have developed compensatory increases in activity in regions associated with attention (i.e., angular gyrus), which may be a protective adaptive mechanism to help maintain an appropriate response to falling glucose levels. However, T1DM patients with hypoglycemia unawareness fail to respond acutely to mild hypoglycemia in cortico-striatal and frontoparietal brain regions. Taken together with the blunted counterregulatory hormone and subjective hypoglycemia symptom responses seen among these individuals, these CNS changes most likely play an important role in causing the inability of T1DM patients with hypoglycemia unawareness to detect and respond appropriately to falling plasma glucose levels. These findings underscore the importance of future interventional studies to determine whether reduction of hypoglycemia frequency can restore these changes in regional brain responses.

\section{Methods}

Participants. Participants were recruited from the greater New Haven area as well as the Yale Diabetes Center. Inclusion criteria for all subjects included ages 18-60 years, BMI less than $30 \mathrm{~kg} / \mathrm{m}^{2}$, and ability to read English. Inclusion criteria for individuals with T1DM included $\mathrm{HbA1c}$ less than $9 \%$ and duration of diabetes more than 5 years. Exclusion criteria included inability to enter the MRI, smoking, illicit drug or recent steroid use, known psychiatric or neurological disorders, active infection, malignancy, abnormal thyroid function, cerebrovascular disease, cardiovascular disease, hepatobiliary disease, weight change in the last 3 months, and pregnancy or breastfeeding.

Sixty-seven potential subjects were screened at the Yale New Haven Hospital Research Unit (HRU) from November 2013 through July 2016 with a screening history, electrocardiogram, physical examination, and laboratory testing at the HRU. Of the 67 subjects screened, 42 participants completed the study and were included in the final analysis (see CONSORT diagram showing the flow of participants in the study, Supplemental Figure 1). They were divided into the following 3 groups: 13 HCs ( 6 males/7 females, age $33 \pm 11$ years, BMI $24.1 \pm$ $\left.2.9 \mathrm{~kg} / \mathrm{m}^{2}, \mathrm{HbA1c} 5.0 \% \pm 0.3 \%\right), 16 \mathrm{~T} 1 \mathrm{DM}$-Aware (5 males/11 females, age $30 \pm 8$ years, BMI $23.2 \pm 3.4 \mathrm{~kg} / \mathrm{m}^{2}, \mathrm{HbA1c} 7.0 \% \pm 0.8 \%$ ), and 13 T1DM-Unaware ( 6 males/7 females, age $40 \pm 12$ years, BMI $26.8 \pm 2.9$ $\mathrm{kg} / \mathrm{m}^{2}, \mathrm{HbA1c} 6.9 \% \pm 0.6 \%$ ). The Clarke score (14) was used to differentiate participants with hypoglycemia awareness versus unawareness. If the Clarke score was not classifiable (i.e., when individuals reported a score of $3 \mathrm{R}[\mathrm{R}=$ number of questions designated "reduced awareness"]), then the Gold (61) method was used to determine whether they had impaired hypoglycemia awareness.

Study protocol. All participants with T1DM were asked to wear a continuous glucose monitor (CGM) (Dexcom G4) 1 week prior to their scheduled MRI visit in order to monitor for antecedent hypoglycemia. If participants had any episodes of hypoglycemia (glucose $<70 \mathrm{mg}$ / $\mathrm{dl}$ or a symptomatic episode requiring assistance) in the 5 days prior to MRI scanning, then the scans were postponed to a later date. On the day of the MRI, participants arrived to the HRU at 9 AM. All participants were instructed to eat breakfast as usual prior to arrival and those with diabetes were further instructed to bolus insulin as usual for breakfast. At $10 \mathrm{AM}$, all participants were provided with a standardized snack consisting of 41 grams of carbohydrate (turkey sandwich, apple, diet ginger ale) in order to neutralize feelings of hunger 
as previously described (5). Participants with diabetes were instructed to inject a bolus of insulin as per their home insulin-to-carbohydrate ratio. Intravenous catheters were placed in antecubital veins bilaterally: one for blood sampling and the other for insulin and glucose infusion. Participants were informed that their glucose levels would be reduced below normal using an insulin and glucose infusion, which could lead to symptoms of hypoglycemia. Participants were blinded to the timing of changes in glucose levels. Scanning began in the MRI center at $12 \mathrm{PM}$ simultaneously with initiation of an insulin infusion at 2 milliunits $/ \mathrm{kg} / \mathrm{h}$. Euglycemia $(\sim 0 \mathrm{mg} / \mathrm{dl})$ was maintained for the first phase of the study, after which glucose was decreased into the mild hypoglycemia range $(\sim 60 \mathrm{mg} / \mathrm{dl})$ (Figure $1 \mathrm{~A})$. BOLD images were acquired during euglycemia (between 45 and 60 minutes) and hypoglycemia (between 90 and 105 minutes) sessions. Participants completed a visual food task while BOLD images were collected, as described below. Throughout the MRI scan, blood was sampled for glucose every 5 minutes. Counterregulatory hormones (epinephrine, norepinephrine, glucagon, and cortisol) were sampled at 0, 30, 45, 60, 75,90 , and 105 minutes.

Biochemical analysis. Plasma glucose was measured enzymatically using glucose oxidase (YSI). Plasma-free insulin, leptin, ghrelin, and glucagon were measured by double-antibody radioimmunoassay (Millipore), as was plasma cortisol (MP Biomedicals). Plasma epinephrine and norepinephrine were measured by high-performance liquid chromatography (ESA).

Visual food cue task. The visual food cue task we used has been previously validated for fMRI $(5,47)$. During each euglycemia and hypoglycemia session, we presented a total of 42 images (3 runs of 14 pictures [7 high-calorie food images, 7 non-food images] each). Highcalorie food pictures included items such as hamburgers, pizza, ice cream, and chocolate as previously described (5). Seventy-five percent of the high-calorie foods were also high-carbohydrate foods. Nonfood pictures consisted of objects such as buildings, books, and doors. Using an event-related design, images were shown for 6 seconds. Each picture was displayed only once and the order of pictures was counterbalanced and randomized within condition across participants. At the end of each trial, a fixation cross appeared with a jittered inter-trial interval (mean, 6 seconds; range, 3-9 seconds), during which participants relaxed until the onset of the next trial, as previously described (5). This process was repeated for each of the 3 runs that were presented at both euglycemia and hypoglycemia.

Hypoglycemia symptom assessments. Participants were asked to verbally rate their sensation of hypoglycemic symptoms (unable to concentrate, blurry vision, anxiety, confusion, difficulty speaking, double vision, drowsiness, tiredness, hunger, weakness, sweating, trembling, warmness, heart racing) on a 7-point Likert scale (1 indicating "not at all" and 7 indicating "a lot") based upon the Edinburgh hypoglycemia symptom score (15) at 3 separate time points during the study: baseline (prior to entering the scanner) and then once they had reached target glucose levels for euglycemia (at 30 minutes) and hypoglycemia (at 90 minutes).

Statistics. One-way analysis of variance (ANOVA) was used to determine whether there were statistical differences among the 3 groups for all demographic variables followed by Fisher's least significant difference (LSD) test for pairwise comparisons if the overall test was statistically significant. Analysis of repeatedly measured variables such as plasma glucose was performed using the mixed- effects regression model method, taking into account both betweensubject and within-subject correlations of repeated measures using a combination of prespecified compound symmetry covariance matrix and an autoregressive covariance matrix. Age, gender, and BMI were adjusted as covariates (i.e., as fixed effects). Subsequently, pairwise comparisons at each time point were performed. Least square mean difference and its $95 \%$ confidence interval are reported as a measure of effect size. To assess changes in counterregulatory hormones, plasma hormone levels at euglycemia (45 and 60 minutes) and hormone levels at hypoglycemia (90 and 105 minutes) were averaged together and compared using paired $t$ tests. All analyses were performed using SAS, version 9.4 and SPSS, version 22 (IBM). A 2-tailed $P$ value of less than 0.05 was considered to be statistically significant. Unless otherwise stated, data are presented as the mean \pm standard error of the mean (SEM).

Study approval. The protocol was approved by the Yale University School of Medicine Human Investigation Committee. All subjects provided informed, written consent before participation.

$f M R I$ analysis. The digital data (Digital Imaging and Communication in Medicine [DICOM]) were converted to NIFTI using dcm2nii (62) and then the first 3 images were discarded from each functional run to enable the signal to achieve steady-state equilibrium between radio-frequency pulsing and relaxation leaving 271 images per slice per run for analysis. The data were motion corrected using SPM8 (www.fil.ion.ucl.ac.uk/spm/software/spm8), and they were discarded if linear motion was greater than $1.5 \mathrm{~mm}$ or rotation was greater than 2 degrees. Images were iteratively smoothed until the smoothness for any image had a full width half maximum of approximately $6 \mathrm{~mm}$ (63). For individual subject data analysis, GLM was used to determine the regions with changes in signal in response to the visual task (highcalorie food or non-food image) in each session. To consider potential variability in baseline fMRI signal, drift correction was included in the GLM with drift regressors used to remove the mean time course, linear, quadratic, and cubic trends for each run. To adjust for anatomical differences in each individual, the Yale Bio-Image Suite software package (http://www.bioimagesuite.org/) was used to calculate 2 linear and 1 nonlinear registration. These 3 registrations were concatenated and applied as 1 registration to bring the data into a common reference brain space. The Colin27 Brain in the Montreal Neurological Institute (MNI) space was used as the reference brain. For group-level data analysis, linear effects modeling using AFNI 3dLME (http://afni. nimh.nih.gov) was implemented with a 3 (group: HC, T1DM-Aware, T1DM-Unaware) $\times 2$ (session: euglycemia, hypoglycemia) $\times 2$ (task: high-calorie food and non-food) design, while covarying for age, duration of diabetes, and BMI using the LME modeling program 3dLME from AFNI (https://afni.nimh.nih.gov/LME). In this design, task and session were treated as the within-subjects fixed-effect factors and group as the between-subjects factor and subject as the random-effect factor. To correct for multiple comparisons, we used FWE correction determined by Monte Carlo simulation using the AFNI 3dClustSim version (16.3.05, October 2016) program. Results are shown at $P$ less than 0.05 whole-brain FWE corrected with an initial $P$ threshold of less than 0.001, as described previously (16).

\section{Author contributions}

JJH and R. Sherwin had full access to all of the data in the study and take responsibility for the integrity of the data and the accu- 
racy of the data analysis. JJH, DS, RTC, R. Sinha, and R. Sherwin conceived and designed the study. JJH, LP, DS, CS, MH, WL, and $\mathrm{RBD}$ acquired the data. $\mathrm{CL}, \mathrm{JJH}, \mathrm{FD}, \mathrm{WL}$, and $\mathrm{MH}$ performed statistical analyses. All authors analyzed and interpreted the data and contributed to writing the manuscript.

\section{Acknowledgments}

This study was supported in part by grants from the NIH R01DK020495 and P30 DK045735 (to R. Sherwin), K23DK109284 (to JJH), and K08AA023545 (to DS). The Yale Center for Clinical Investigation was supported by an NIH Clinical Translational Sci- ence Award (UL1 RR024139). We gratefully acknowledge the help of the Yale Core lab staff: Mikhail Smolgovsky, Irene Chernyak, Ralph Jacob, Doreen Nemeth, Maria Batsu, and Codruta Todeasa; as well as the Yale HRU nurses and staff: Joanne Caprio-Adams, Gina Solomon, Anne O'Connor, Catherine Parmelee, Mary Scanlon, Lynda Knaggs, Carmen Galarza, Elizabeth O’Neal, Joyce Russell, Gayle Pietrogallo, and Cynthia Smith.

Address correspondence to: Robert S. Sherwin, The Anlyan Center, TAC 147S, PO Box 208020, New Haven, Connecticut 06520, USA. Phone: 203.785.4183; Email: robert.sherwin@yale.edu.
1. DIAMOND Project Group. Incidence and trends of childhood Type 1 diabetes worldwide 19901999. Diabet Med. 2006;23(8):857-866.

2. Cryer PE. Banting Lecture. Hypoglycemia: the limiting factor in the management of IDDM. Diabetes. 1994;43(11):1378-1389.

3. Sherwin RS. Bringing light to the dark side of insulin: a journey across the blood-brain barrier. Diabetes. 2008;57(9):2259-2268.

4. Page KA, Arora J, Qiu M, Relwani R, Constable RT, Sherwin RS. Small decrements in systemic glucose provoke increases in hypothalamic blood flow prior to the release of counterregulatory hormones. Diabetes. 2009;58(2):448-452.

5. Page KA, et al. Circulating glucose levels modulate neural control of desire for high-calorie foods in humans. J Clin Invest. 2011;121(10):4161-4169.

6. Gerich JE, Langlois M, Noacco C, Karam JH, Forsham PH. Lack of glucagon response to hypoglycemia in diabetes: evidence for an intrinsic pancreatic alpha cell defect. Science. 1973;182(4108):171-173.

7. Bolli G, et al. Abnormal glucose counterregulation in insulin-dependent diabetes mellitus. Interaction of anti-insulin antibodies and impaired glucagon and epinephrine secretion. Diabetes. 1983;32(2):134-141.

8. Hirsch BR, Shamoon H. Defective epinephrine and growth hormone responses in type I diabetes are stimulus specific. Diabetes. 1987;36(1):20-26.

9. Cryer PE, Fisher JN, Shamoon H. Hypoglycemia. Diabetes Care. 1994;17(7):734-755.

10. White NH, Skor DA, Cryer PE, Levandoski LA, Bier DM, Santiago JV. Identification of type I diabetic patients at increased risk for hypoglycemia during intensive therapy. $N$ Engl JMed. 1983;308(9):485-491.

11. Bolo NR, et al. Functional connectivity of insula, basal ganglia, and prefrontal executive control networks during hypoglycemia in type 1 diabetes. J Neurosci. 2015;35(31):11012-11023.

12. Dunn JT, Cranston I, Marsden PK, Amiel SA, Reed LJ. Attenuation of amydgala and frontal cortical responses to low blood glucose concentration in asymptomatic hypoglycemia in type 1 diabetes: a new player in hypoglycemia unawareness? Diabetes. 2007;56(11):2766-2773.

13. Suda S, Shinohara M, Miyaoka M, Lucignani G, Kennedy C, Sokoloff L. The lumped constant of the deoxyglucose method in hypoglycemia: effects of moderate hypoglycemia on local cerebral glucose utilization in the rat. JCereb Blood Flow Metab. 1990;10(4):499-509.
14. Clarke WL, Cox DJ, Gonder-Frederick LA, Julian D, Schlundt D, Polonsky W. Reduced awareness of hypoglycemia in adults with IDDM. A prospective study of hypoglycemic frequency and associated symptoms. Diabetes Care. 1995;18(4):517-522.

15. Deary IJ, Hepburn DA, MacLeod KM, Frier BM. Partitioning the symptoms of hypoglycaemia using multi-sample confirmatory factor analysis. Diabetologia. 1993;36(8):771-777.

16. Cox RW, Chen G, Glen DR, Reynolds RC, Taylor PA. FMRI Clustering in AFNI: false-positive rates redux. Brain Connect. 2017;7(3):152-171.

17. Grahn JA, Parkinson JA, Owen AM. The role of the basal ganglia in learning and memory: neuropsychological studies. Behav Brain Res. 2009;199(1):53-60.

18. Grahn JA, Parkinson JA, Owen AM. The cognitive functions of the caudate nucleus. Prog Neurobiol. 2008;86(3):141-155.

19. Yin HH, Knowlton BJ, Balleine BW. Blockade of NMDA receptors in the dorsomedial striatum prevents action-outcome learning in instrumental conditioning. Eur J Neurosci. 2005;22(2):505-512.

20. Yin HH, Knowlton BJ, Balleine BW. Lesions of dorsolateral striatum preserve outcome expectancy but disrupt habit formation in instrumental learning. Eur J Neurosci. 2004;19(1):181-189.

21. Tricomi EM, Delgado MR, Fiez JA. Modulation of caudate activity by action contingency. Neuron . 2004;41(2):281-292.

22. Lehéricy S, et al. Diffusion tensor fiber tracking shows distinct corticostriatal circuits in humans. Ann Neurol. 2004;55(4):522-529.

23. Alexander GE, DeLong MR, Strick PL. Parallel organization of functionally segregated circuits linking basal ganglia and cortex. Annu Rev Neurosci. 1986;9:357-381.

24. Teh MM, et al. Evolution and resolution of human brain perfusion responses to the stress of induced hypoglycemia. Neuroimage. 2010;53(2):584-592.

25. Musen $\mathrm{G}$, et al. Regional brain activation during hypoglycemia in type 1 diabetes. J Clin Endocrinol Metab. 2008;93(4):1450-1457.

26. Sakurai Y. [Brodmann areas 39 and 40: human parietal association area and higher cortical function]. Brain Nerve. 2017;69(4):461-469.

27. Ptak R, Schnider A. The attention network of the human brain: relating structural damage associated with spatial neglect to functional imaging correlates of spatial attention. Neuropsychologia. 2011;49(11):3063-3070.

28. Ptak R. The frontoparietal attention network of the human brain: action, saliency, and a prior- ity map of the environment. Neuroscientist. 2012;18(5):502-515.

29. Jung RE, Haier RJ. The parieto-frontal integration theory (P-FIT) of intelligence: converging neuroimaging evidence. Behav Brain Sci. 2007;30(2):135-154.

30. Corbetta M, Shulman GL. Human cortical mechanisms of visual attention during orienting and search. Philos Trans R Soc Lond, B, Biol Sci. 1998;353(1373):1353-1362.

31. Corbetta M. Frontoparietal cortical networks for directing attention and the eye to visual locations: identical, independent, or overlapping neural systems? Proc Natl Acad Sci U S A. 1998;95(3):831-838.

32. Gottlieb J. From thought to action: the parietal cortex as a bridge between perception, action, and cognition. Neuron. 2007;53(1):9-16.

33. Singh-Curry V, Husain M. The functional role of the inferior parietal lobe in the dorsal and ventral stream dichotomy. Neuropsychologia. 2009;47(6):1434-1448.

34. Rosenthal JM, et al. The effect of acute hypoglycemia on brain function and activation: a functional magnetic resonance imaging study. Diabetes. 2001;50(7):1618-1626.

35. Jeong SK, Xu Y. The impact of top-down spatial attention on laterality and hemispheric asymmetry in the human parietal cortex. J Vis. 2016;16(10):2.

36. Grill V, et al. Cerebral blood flow and substrate utilization in insulin-treated diabetic subjects. Am J Physiol. 1990;258(5 Pt 1):E813-E820.

37. Gulanski BI, et al. Increased brain transport and metabolism of acetate in hypoglycemia unawareness. J Clin Endocrinol Metab. 2013;98(9):3811-3820.

38. Mangia S, et al. Hypoglycemia-induced increases in thalamic cerebral blood flow are blunted in subjects with type 1 diabetes and hypoglycemia unawareness. J Cereb Blood Flow Metab. 2012;32(11):2084-2090.

39. Wiegers EC, et al. Cerebral blood flow response to hypoglycemia is altered in patients with type 1 diabetes and impaired awareness of hypoglycemia. JCereb Blood Flow Metab. 2017;37(6):1994-2001.

40. Simpson IA, et al. Blood-brain barrier glucose transporter: effects of hypo- and hyperglycemia revisited. J Neurochem. 1999;72(1):238-247.

41. Kumagai AK, Kang YS, Boado RJ, Pardridge WM. Upregulation of blood-brain barrier GLUT1 glucose transporter protein and mRNA in experimental chronic hypoglycemia. Diabetes. 
1995;44(12):1399-1404.

42. Bolo NR, et al. Brain activation during working memory is altered in patients with type 1 diabetes during hypoglycemia. Diabetes. 2011;60(12):3256-3264.

43. van de Ven KC, van der Graaf M, Tack CJ, Heerschap A, de Galan BE. Steady-state brain glucose concentrations during hypoglycemia in healthy humans and patients with type 1 diabetes. Diabetes. 2012;61(8):1974-1977.

44. Dagogo-Jack S, Fanelli CG, Cryer PE. Durable reversal of hypoglycemia unawareness in type 1 diabetes. Diabetes Care. 1999;22(5):866-867.

45. Dagogo-Jack S, Rattarasarn C, Cryer PE. Reversal of hypoglycemia unawareness, but not defective glucose counterregulation, in IDDM. Diabetes. 1994;43(12):1426-1434.

46. Fanelli C, et al. Long-term recovery from unawareness, deficient counterregulation and lack of cognitive dysfunction during hypoglycaemia, following institution of rational, intensive insulin therapy in IDDM. Diabetologia. 1994;37(12):1265-1276.

47. Belfort-DeAguiar R, et al. Food image-induced brain activation is not diminished by insulin infusion. Int JObes (Lond). 2016;40(11):1679-1686.

48. Rushworth MF, Noonan MP, Boorman ED, Walton ME, Behrens TE. Frontal cortex and rewardguided learning and decision-making. Neuron.
2011;70(6):1054-1069.

49. Schoenbaum G, Roesch MR, Stalnaker TA, Takahashi YK. A new perspective on the role of the orbitofrontal cortex in adaptive behaviour. Nat Rev Neurosci. 2009;10(12):885-892.

50. Hirose $S$, et al. Lateral-medial dissociation in orbitofrontal cortex-hypothalamus connectivity. Front Hum Neurosci. 2016;10:244.

51. Barbas H. Flow of information for emotions through temporal and orbitofrontal pathways. JAnat. 2007;211(2):237-249.

52. Rolls ET, Baylis LL. Gustatory, olfactory, and visual convergence within the primate orbitofrontal cortex. J Neurosci. 1994;14(9):5437-5452.

53. Rolls ET, Verhagen JV, Kadohisa M. Representations of the texture of food in the primate orbitofrontal cortex: neurons responding to viscosity, grittiness, and capsaicin. J Neurophysiol. 2003;90(6):3711-3724.

54. O'Doherty J, et al. Sensory-specific satiety-related olfactory activation of the human orbitofrontal cortex. Neuroreport. 2000;11(4):893-897.

55. Ortiz-Alonso FJ, Galecki A, Herman WH, Smith MJ, Jacquez JA, Halter JB. Hypoglycemia counterregulation in elderly humans: relationship to glucose levels. Am J Physiol.1994; 267(4 Pt 1):E497-E506.

56. Meneilly GS, Minaker KL, Young JB, Landsberg L, Rowe JW. Counterregulatory responses to insulin-induced glucose reduction in the elderly. J Clin Endocrinol Metab. 1985;61(1):178-182.

57. Nunley KA, et al. White matter hyperintensities in middle-aged adults with childhood-onset type 1 diabetes. Neurology. 2015;84(20):2062-2069.

58. Biessels GJ, Reijmer YD. Brain changes underlying cognitive dysfunction in diabetes: what can we learn from MRI? Diabetes. 2014;63(7):2244-2252.

59. Brands AM, et al. Cognitive performance, psychological well-being, and brain magnetic resonance imaging in older patients with type 1 diabetes. Diabetes. 2006;55(6):1800-1806.

60. Simmons JP, Nelson LD, Simonsohn U. Falsepositive psychology: undisclosed flexibility in data collection and analysis allows presenting anything as significant. Psychol Sci. 2011;22(11):1359-1366.

61. Gold AE, MacLeod KM, Frier BM. Frequency of severe hypoglycemia in patients with type I diabetes with impaired awareness of hypoglycemia. Diabetes Care. 1994;17(7):697-703.

62. Li X, Morgan PS, Ashburner J, Smith J, Rorden C. The first step for neuroimaging data analysis: DICOM to NIfTI conversion. J Neurosci Methods. 2016;264:47-56.

63. Friedman L, Glover GH, FBIRN Consortium. Reducing interscanner variability of activation in a multicenter fMRI study: controlling for signalto-fluctuation-noise-ratio (SFNR) differences. Neuroimage. 2006;33(2):471-481. 